\title{
IMPACTO DEL MICROCRÉDITO SOBRE LA POBREZA RURAL EN LOS MUNICIPIOS DE TUNJA Y SAMACÁ, COLOMBIA*
}

\author{
DOI: $10.22395 /$ seec.v20n45a2
Gloria Manrique Joya**
Maricela Ramírez ${ }^{* * *}$ \\ Félix Santos Varón ${ }^{* * * *}$
}

Recibido: 15 de diciembre de 2016 - Aprobado: 27 de septiembre de 2017

\section{RESUMEN}

El objetivo de este artículo es evaluar el efecto de los microcréditos rurales sobre la pobreza, a través de cambios en la dimensión económica: ingresos, activos fijos y activos productivos. Se usa el método de emparejamiento (Propensity Score Matching) entre beneficiarios y no beneficiarios de los microcréditos del Banco Agrario en los municipios de Tunja y Samacá (Boyacá, Colombia). Los resultados dan cuenta de impactos positivos que sugieren que el microcrédito ha contribuido a la acumulación de capital, lo que podría contribuir a que, en el mediano o largo plazo, se mejoren las condiciones de vida y se reduzcan los niveles de pobreza del grupo de beneficiarios de los microcréditos.

\section{PALABRAS CLAVE}

Crédito agrícola; microcrédito; pobreza; ingresos; Colombia.

\section{CLASIFICACION JEL:}

I30; I32; Q14

\section{CONTENIDO:}

Introducción; 1. Revisión literaria; 2. Metodología; 3. Discusión y resultados; 4. Conclusiones y recomendaciones; Bibliografía.

Artículo de investigación derivado del estudio "Microfinanzas rurales y sus contribuciones en la reducción de la pobreza rural en Tunja y áreas de influencia"; adscrito al Grupo de Investigación Dinámica Contable (GIDICON, clasificado por Colciencias en categoría C en 2017). Este proyecto fue financiado por Facultad de Ciencias Económicas y Administrativas de la Universidad Pedagógica y Tecnológica de Colombia, fue desarrollado en el período 2014-2016 y contó con el valioso apoyo del Banco Agrario (Seccional Tunja).

** Economista, Universidad Pedagógica y Tecnológica de Colombia, Tunja, Colombia. Magíster en Economía, Universidad Pedagógica y Tecnológica de Colombia, Tunja, Colombia. Magíster en Dirección y Asesoramiento Financiero, Universidad Internacional de la Rioja, Logroño, España. Especialista en Finanzas, Universidad Antonio Nariño. Bogotá, Colombia. Docente e Investigadora, Universidad Pedagógica y Tecnológica de Colombia, sede central, Tunja, Colombia. Miembro activo del grupo de investigación GIDICON. Dirección: Avenida Central del Norte 39-115, Teléfono 3103055470. Correo electrónico: gloriamercedes.manrique@uptc.edu.co

*** Contadora Pública, Universidad Pedagógica y Tecnológica de Colombia, Sogamoso, Colombia. Magíster en Administración Económica y Financiera, Universidad Tecnológica de Pereira, Pereira, Colombia. Especialista en Revisoría Fiscal, Universidad Jorge Tadeo Lozano, Colombia. Docente Investigadora Universidad Pedagógica y Tecnológica de Colombia, sede central, Tunja, Colombia. Miembro activo del grupo de investigación GIDICON. Dirección: Avenida Central del Norte 39-115, Teléfono 3204878826. Correo electrónico: maricela.ramirez@uptc.edu.co

*** Contador público, Universidad Libre, Bogotá, Colombia. Magíster en Educación, Universidad Javeriana, Bogotá, Colombia. Magíster en Administración, Universidad Santo Tomás, Bucaramanga, Colombia. Especialista en Revisoría Fiscal y Auditoria, Universidad Externado, Bucaramanga, Colombia. Docente e Investigador, Universidad Pedagógica y Tecnológica de Colombia, sede central, Tunja, Colombia. Dirección: Avenida Central del Norte 39-115, Teléfono 3204878826. Correo electrónico: felix.varon@uptc.edu.co 


\title{
IMPACT OF MICROCREDIT ON RURAL POVERTY IN MUNICIPALITIES OF TUNJA AND SAMACÁ, COLOMBIA
}

\begin{abstract}
The goal of this article is to assess the impact of rural microcredits on poverty through changes in the economic dimension: income, fixed assets, and productive assets. The method of matching (Propensity Score Matching) is used between beneficiaries and non-beneficiaries of Banco Agrario microcredits in the Tunja and Samacá municipalities (Boyacá, Colombia). The results show positive impacts suggesting that the microcredit has contributed to the accumulation of capital, which could contribute to improve living conditions and reduce poverty levels in the microcredit beneficiary group at the middle or long term.
\end{abstract}

\section{KEYWORDS}

Agricultural credit; microcredit; poverty; income; Colombia.

\section{JEL CLASSIFICATION}

I30; I32; Q14

\section{CONTENT}

Introduction; 1. Literary review; 2. Methodology; 3 . Discussion and Results; 4. Conclusions and Recommendations; Bibliography.

\section{IMPACTO DO MICROCRÉDITO SOBRE A POBREZA RURAL NOS MUNICÍPIOS DE TUNJA E SAMACÁ, COLÔMBIA}

\section{RESUMO}

O objetivo deste artigo é avaliar o efeito dos microcréditos rurais sobre a pobreza por meio de mudanças na dimensão econômica: ingressos, ativos fixos e ativos produtivos. Utiliza-se o escore de propensão (Propensity Score Matching) entre beneficiários e não beneficiários dos microcréditos do Banco Agrario nos municípios de Tunja e Samacá (estado de Boyacá, Colômbia). Os resultados demonstram impactos positivos que sugerem que o microcrédito contribua para a acumulação de capital, o que poderia corroborar a que, no médio ou longo prazo, sejam melhoradas as condições de vida e reduzidos os níveis de pobreza do grupo de beneficiários dos microcréditos.

\section{PALAVRAS-CHAVE}

Crédito agrícola; microcrédito; pobreza; ingressos; Colômbia.

\section{CLASSIFICAÇÃO JEL}

\author{
I30; I32; Q14
}

\section{CONTEÚDO}

Introdução; 1. Revisão da literatura; 2. Metodologia; 3. Discussão e resultados; 4. Conclusões e recomendações; Bibliografia. 


\section{INTRODUCCIÓN}

Aunque existe amplia literatura sobre los aportes de los microcréditos en la reducción de la pobreza, son escasos los estudios que con una metodología apropiada pueden probar el efecto de los pequeños créditos en las mejoras del desempeño económico y social de las familias beneficiarias.

Como se cita en Armendariz y Morduch (2005), analizar el impacto del financiamiento en poblaciones de escasos recursos puede resultar costoso y, dada la proliferación de instituciones dedicadas a conceder microcréditos, se asume de manera errática que un aumento de la oferta implica impactos de tipo positivo en los beneficiarios, por lo que se requiere de trabajos cuantitativos que basados en datos y metodologías apropiadas puedan probar su validez.

En Cotler y Rodríguez-Oreggia (2010), con el uso de una técnica rigurosa de evaluación cuasi experimental como el Propensity Score Matching (PSM), se miden los efectos de los pequeños créditos sobre los activos no financieros como indicador de bienestar de los hogares en México.

Para Colombia, aunque los programas de microfinanciamiento han sido centro de la política pública y cada día la oferta por parte de las instituciones financieras viene en aumento, son pocas las investigaciones dedicadas a revisar los verdaderos efectos del microcrédito en las poblaciones rurales donde el índice de pobreza indica mayor concentración.

Este trabajo aplica un análisis estadístico con el PSM para medir el impacto del microcrédito en poblaciones rurales. El artículo está organizado de la siguiente manera: en la primera parte se realiza una revisión teórica sobre investigaciones que involucran causalidad, enseguida se describe la metodología utilizada exponiendo el procedimiento cuasi experimental para calcular los efectos del microcrédito sobre los individuos beneficiarios y, por último, se ilustran los resultados obtenidos, y se presentan conclusiones y limitaciones.

\section{REVISIÓN LITERARIA}

El crecimiento económico se considera necesario, pero no suficiente para mitigar la pobreza; en ocasiones el efecto positivo que este produce en el bienestar puede minimizarse o inclusive anularse debido a una inequitativa distribución del ingreso. Políticas de tipo macroeconómico top-down ${ }^{1}$ requieren acompañarse de políti-

Enfoque denominado de "arriba hacia abajo" hace referencia a la teoría del crecimiento local con políticas que parten del nivel Estatal hacia territorios necesitados de desarrollo. 
cas microeconómicas bottom-up bien diseñadas, que favorezcan el incremento de la productividad y la participación de las personas menos favorecidas en el mercado como lo señalan Snow y Terry (2001) y Woller, Dunford y Woodworth (1999).

En este sentido el microcrédito se ha convertido en una estrategia para el alivio de la pobreza. Con la creación del Banco Grameen se promueve la inserción al mercado del crédito a personas excluidas del sector formal a bajas tasas de interés y el depósito de confianza en el deudor para promover la productividad y el ahorro. Desde la vía financiera como lo señala Yunus (2010) se puede dotar de pequeñas unidades de capital (microcrédito) a una persona para desarrollar su creatividad y lograr que la pobreza desaparezca de forma sistemática y rápida.

El éxito del Banco Grameen y el auge de los programas de microcrédito alrededor del mundo despertaron el interés por su evaluación, utilizando técnicas cuasi experimentales como PSM.

Morduch (1998), Hossain(1988), Gibbons y Garfield (1999), Hulme y Mosley (1996), Copestake et al. (2005), Arun, Imai y Sinha (2006), Lacalle, Rico y Durán (2008) y Karlan y Zinman (2007) evidenciaron que para cada contexto de análisis los impactos son diversos y están asociados a la efectividad del microcrédito en las dimensiones económicas y sociales de los hogares beneficiarios.

Khandker (1998) examina el crédito en Bangladesh y encuentra impacto significativo sobre el ingreso, estabilización del consumo, regulación en la mano de obra y una reducción del $5 \%$ en el nivel de pobreza sobre las familias participantes, pero advierte que los efectos solo serán sostenibles si el microcrédito se orienta a zonas donde existe potencial de crecimiento.

MkNelly y Dunford (1998) con estudios en la zona costera de Ghana encontraron que pequeñas unidades de préstamo del Banco de Crédito para la educación CRECER $^{3}$ provocaron mejores condiciones en el hogar, medidas por cambios en las actividades económicas y empoderamiento de las mujeres para alcanzar seguridad alimentaria. Igualmente, MkNelly y Dunford (1999), al evaluar los impactos de este mismo préstamo en Bolivia, comprobaron aumentos en los ingresos, los ahorros y las condiciones de salud en los participantes.

2 Enfoque denominado de "abajo hacia arriba" se refiere a la teoría del crecimiento local con políticas que van encaminadas a trabajar de la mano con la comunidad.

3 Crédito con Educación Rural. Creado para brindar seguridad alimentaria y bienestar a familias y comunidades complementado con servicios educativos de alta calidad y accesibles para mujeres que viven en áreas rurales. 
Zaman (1999) pudo establecer que el microcrédito produjo una reducción de la vulnerabilidad a través de mayores y más diversificados ingresos ${ }^{4}$, consumo permanente en el tiempo, construcción de bienes duraderos, acceso a asistencia de emergencia durante desastres naturales y empoderamiento en las mujeres de Bangladesh.

Armendariz y Morduch (2005) discuten indicadores de productividad, y rentabilidad, y el alcance en la reducción de pobreza de los clientes del pequeño crédito. Los autores aclaran que las mediciones tienen complejidades y los estudios pueden tornarse, desde la metodología, poco sólidos, en el sentido en que las condiciones iniciales que engendran la pobreza en determinados individuos es diferente y la forma como se genera la muestra y los datos de los cuales se desprende la medición son fiables en la medida en que se controlen los problemas de autoselección o de endogeneidad y se den similitudes entre los beneficiarios y no beneficiarios del programa.

Morán y Guerrero (2009) revelan que los microcréditos concedidos por la Corporación Hogar de Cristo de Guayaquil (Ecuador) a mujeres con ideas de negocios en las provincias de Pichincha, Azuay y Guayasen, aunque no presentaron impactos en el ingreso per cápita, si ocasionaron efectos positivos en el empoderamiento.

Y, en Álvarez (2013) los impactos del microcrédito otorgado por la Cooperativa Colanta en Colombia evidencian una relación positiva entre la situación económica y la social de sus asociados, a través de mejores ingreso y mayores posibilidades de ahorro.

\section{METODOLOGÍA}

\subsection{Aproximaciones conceptuales del Propensity Score Matching (PSM)}

Para evaluar el impacto de los microcréditos rurales como herramienta de alivio a la pobreza, a través de cambios en la dimensión económica: ingresos, activos fijos y activos productivos de las personas beneficiarias del microcrédito del Banco Agrario (seccional Tunja-Colombia), se usó una técnica estadística de medición de impacto tipo cuasi experimental PSM basada en el cálculo de un escenario contrafactual en ausencia de intervención que permite aproximar el efecto causal de la misma. Para su estimación se constituyen dos grupos de comparación: el grupo de tratamiento (GT), conformado por quienes son intervenidos o son beneficiarios del programa evaluado (personas con microcrédito), y el grupo de control (GC), confor-

\footnotetext{
${ }^{4}$ A través del fomento de actividades productivas en pequeños empresarios como cría de aves y siembra de patatas, entre otras.
} 
mado por quienes pueden ser elegibles con características económicas, sociales y geográficas similares al grupo de beneficiarios pero que no son intervenidos o no son beneficiarios del programa (personas que no han accedido al microcrédito).

El PSM estima el estado de los beneficiarios de un programa en ausencia de la intervención, es decir el efecto neto causado por la diferencia entre quienes son beneficiarios del microcrédito y quienes no lo han sido, medido sobre las variables resultados que se identifican como aquellas sobre las cuales se espera que el microcrédito haya causado algún efecto en los individuos beneficiarios como indican Gertler et al. (2011). Para el caso, corresponden a las variables binarias reflejadas en el gráfico 1, que recogen información sobre cambios en los ingresos, mejoras en los activos fijos y productivos de los beneficiarios agrupados en el índex econo5.

\section{Gráfico 1. Variables incorporadas en los cálculos del índex-econo (activos fijos, activos materiales productivos)}

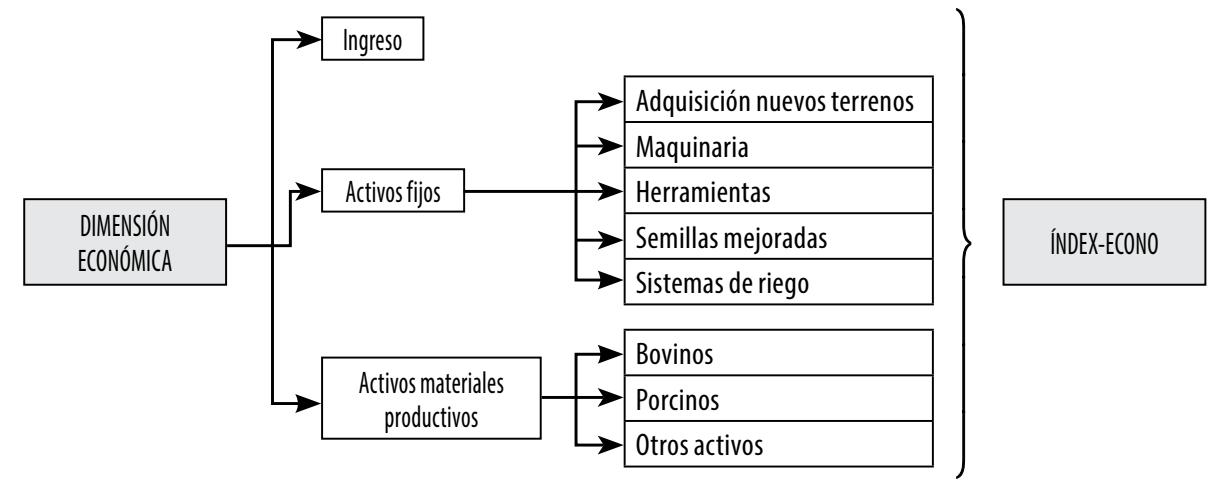

Fuente: elaboración propia

En metodologías como el PSM, determinar el efecto neto presenta limitaciones, como se muestra en Tamayo (2012): la primera limitación surge por la dificultad de observar la situación en que se encuentran los beneficiarios en el escenario de ausencia de la intervención; la segunda obedece a la diferencia que existe entre beneficiarios y controles en ausencia de la intervención y, por último, se da la posibilidad de la existencia de eventos presentes en la intervención que pueden afectar las variables resultado.

De la primera limitante se desprende el problema fundamental de la evaluación de impacto, referido al efecto neto, el cual es definido como la diferencia medible

\footnotetext{
5 Representa un índice que recoge la información en cuanto a activos fijos y productivos de los participantes en el estudio, fue construido con el método de análisis de componentes principales (ACP).
} 
entre la variable resultado $(Y)$ del individuo (i) cuando ha sido tratado $\left(D_{i}=1\right.$, beneficiario del microcrédito) y la variable resultado (Y) del mismo individuo cuando no ha recibido tratamiento $\left(D_{i}=0\right.$, no beneficiario del microcrédito); por tanto, el efecto neto es definido, como:

$$
\mathcal{T}_{i t}=\mathrm{E}\left[\mathrm{Y}_{i t}(\mathrm{D}=1)\right]-\mathrm{E}\left[\mathrm{Y}_{i t}(\mathrm{D}=0)\right]
$$

La anterior ecuación compara al mismo individuo beneficiario del microcrédito en un mismo momento del tiempo, situación que no es posible, ya que solo puede obtenerse uno de los dos resultados, cuando ha sido o cuando no ha sido beneficiario del microcrédito. Es por ello que, de acuerdo con Rosenbaum y Rubin (1983), se debe emplear una metodología de escenario contrafactual, centrado en el cálculo del efecto medio del tratamiento sobre los tratados (average treatment effect on the treated -ATT-); de esta forma el efecto neto de la intervención sería:

$$
\left.\mathcal{T}_{i t}=\mathrm{E}\left[\mathrm{Y}_{i}(1) \mathrm{D}=1\right]-\mathrm{E}\left[\mathrm{Y}_{i}(0) \mathrm{D}=1\right]+\mathrm{E}\left[\mathrm{Y}_{i}(0) \mathrm{D}=1\right]-\mathrm{E}\left[\mathrm{Y}_{i}(0) \mathrm{D}=0\right)\right]
$$

El ATT, en la ecuación [3] se determina por los dos términos del lado derecho de la ecuación [2] y significa la diferencia entre medias de la variable resultado, estudiada con microcrédito o sin microcrédito del GT usando el escenario contrafactual, efecto cuyo éxito en la estimación está condicionado a la confiabilidad del grupo con el que se pretende estimar el contrafactual, es decir, el GC escogido:

$$
\left.\mathrm{ATT}=\mathrm{E}\left|\mathcal{T}_{i}\right| \mathrm{D}_{i}=1\right)=\mathrm{E}\left[\mathrm{Y}_{i}(1) \mid \mathrm{D}_{i}=1\right]-\mathrm{E}\left[\mathrm{Y}_{i}(0) \mid \mathrm{D}_{i}=1\right]
$$

El ATT tiene como limitante que tanto beneficiarios como no beneficiarios del microcrédito son diferentes aun en ausencia del programa, lo que, por supuesto reconoce en este tipo de metodologías cuasi experimentales sesgo de selección, derivado de la no aleatoriedad en la conformación de los grupos tratados, y control como se indica en la ecuación [4]:

$$
\mathrm{E} \mathcal{T}_{i}=\mathrm{ATT}+\underbrace{\mathrm{E}\left[\mathrm{Y}_{i}(0) \mid \mathrm{D}_{i}=1\right]-\mathrm{E}\left[\mathrm{Y}_{i}(0)\left|\mathrm{Y}_{i}(0)\right| \mathrm{D}_{i}=1\right]-\mathrm{E}\left[\mathrm{Y}_{i}(0) \mid\right]}_{\text {Sesgo de selección }}
$$

Asumir que el sesgo de selección se anula es un supuesto demasiado fuerte de cumplir; encontrar estimaciones consistentes e insesgadas aún con la presencia del sesgo es un reto. Para este propósito la metodología cuasi experimental escogida sugiere el supuesto de independencia condicional y la técnica de balanceo. La existencia de características observables y no observables en el término de error que explica el recibir tratamiento (microcrédito) y las variables resultado podrían suponer que la variable tratamiento $\mathrm{D}_{\mathrm{i}}$ podría estar correlacionada con el error $\mathrm{U}_{\mathrm{i}}$; 
por tanto, el resultado del efecto podría estar subestimado o sobreestimado por los no observables como se argumenta en Tamayo (2012). Esta condición obliga a asumir ciertas consideraciones como la generación de un GC (no beneficiarios de microcrédito) muy depurado con características económicas, sociales y geográficas muy similares a los del GT (beneficiarios del microcrédito) con el fin de encontrar mediciones creíbles sobre las variables resultado.

\subsection{Precisiones sobre las variables resultado: construcción del índex-econo}

En la recolección de datos, como lo afirman Filmer y Pritchett (2001), se pueden presentar problemas de dimensionalidad ocasionados por la cantidad de variables que se pretenden involucrar en la medición, por lo que se aconseja la construcción de índices utilizando el método de análisis de componentes principales (ACP), bajo el cual se agregan variables binarias en una sola para distribuir de manera más precisa los pesos de estas en las mediciones.

Para el índex-econo, se utilizó la información recogida en la encuesta sobre los activos fijos y productivos (descritos en la tabla 1). De acuerdo con Carreño, Hernández y Méndez (2011) la estimación del índice se realiza con base en el primer componente principal que incluyen $\left.\left\{z_{1}, z_{2}, \ldots . ., z_{n}\right\}\right\}$ estandarizados ${ }^{6}$, e identificadas como el conjunto de características cuantitativas que se pretenden resumir; entonces el ACP, será:

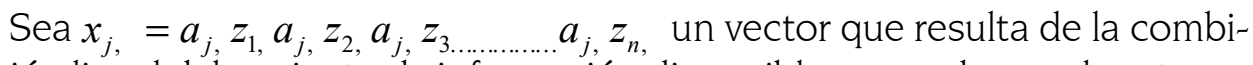
nación lineal del conjunto de información disponible generada por el vector $\boldsymbol{a}_{i}$ :

$$
\begin{gathered}
\boldsymbol{a}_{\boldsymbol{j}}=\left|\begin{array}{c}
a_{j 1} \\
a_{j 2} \\
a_{j 3} \\
\ldots \\
a_{j n}
\end{array}\right| \\
\boldsymbol{z}^{\prime}=\left\{z_{1,} z_{2,} z_{3, \ldots \ldots \ldots \ldots \ldots \ldots} z_{n}\right\} \\
\chi_{i}=a^{\prime}{ }_{i}^{*} z
\end{gathered}
$$

Ecuación [5], utilizada para encontrar el componente principal, maximizando la varianza del componente:

$$
\operatorname{Max} \operatorname{Var}\left(x_{i}\right)=a^{\prime}{ }_{j}^{*} a_{j}=1
$$

${ }_{6}$ Las variables $\boldsymbol{z}^{\prime}=\left\{z_{1}, z_{2}, z_{3}\right.$ $\left.z_{n}\right\}$ deben tener media cero y varianza uno. 
En la ecuación [6], el propósito de la restricción $a^{\prime}{ }_{j}^{*} a_{j}=1$ es encontrar una solución única. De otra parte, la varianza del componente es la varianza de la combinación lineal $a_{j}{ }_{j}^{*} z$, luego:

$$
\begin{gathered}
\operatorname{Var}\left(x_{i}\right)=\operatorname{Var}_{i}{ }^{*} z \\
\operatorname{Var}\left(x_{i}\right)=a_{j}^{\prime}{ }^{*} \sum a_{j} \\
\Sigma \equiv \mathrm{E}(\mathrm{ZZ})
\end{gathered}
$$

Por tanto, la estandarización de Z y $\Sigma$ se identifica como la matriz de correlaciones de Z; de esta forma el cálculo del componente principal se puede reescribir así:

$$
\operatorname{Max} a_{j}^{\prime *} \Sigma a_{j} ; a_{j}^{\prime *} a_{j}=1
$$

Y la solución del problema de maximización por el sistema de ecuación lineal sería:

$$
(\Sigma-\lambda I) a_{j}=0
$$

Para que este sistema tenga solución distinta de cero, se requiere que el determinante sea:

$$
|\Sigma-\lambda I|=0
$$

De esta manera a través de la ecuación [7], se obtienen los eigenvalores $\left\{\lambda_{1}, \lambda_{2}, \lambda_{3} \ldots \ldots . \lambda_{n}\right\}$, de la matriz $\Sigma$, demostrando que la varianza del componente resulta igual a este y da origen al $a_{j}$ :

$$
\operatorname{Var}\left(x_{i}\right)=\lambda
$$

Significando con ello que se toma el eingenvalor más grande $\lambda_{1}$ y se halla aquel en el que la combinación lineal de las variables originales tenga la mayor varianza.

\subsection{Datos}

La muestra utilizada en el estudio es focalizada (no aleatoria); en concordancia con lo indicado en Campbell y Stanley (1995), se conformó con solicitantes y beneficiarios del microcrédito del Banco Agrario ubicados en los municipios de Tunja y Samacá, Colombia.

Los datos fueron generados por los investigadores en los años 2015-2016 a través de encuesta directa a 134 personas (ver formato de encuesta utilizado en anexo) de las cuales solo se incorporaron 128 al análisis, seis de ellas resultaron ser observaciones sin contrafactual, es decir, no se halló parámetro com- 
parable entre GT y GC. La muestra se extrae de los municipios de Tunja y Samacá (Boyacá) dado que son los lugares donde se concentra la colocación del microcrédito analizado.

El GC lo integraron personas no beneficiarias de microcrédito con características socioeconómicas similares a las de los beneficiarios y habitantes de la misma zona geográfica, quienes en alguna oportunidad realizaron solicitud de crédito al banco, pero les fue negada o no hicieron uso del mismo por diversas razones entre las que se encuentra el no estar de acuerdo con la tasa de interés ofertada.

La conformación del GT se realizó con beneficiarios del sistema de microcréditos rurales del Banco Agrario en los municipios de Tunja y Samacá, los cuales son resultado de la política pública de microfinanciamiento para personas naturales y unidades familiares rurales de bajos ingresos y con un potencial de respaldo de deuda restringido. Son créditos concedidos en montos pequeños que van desde 1 a $25 \mathrm{SMMLV}^{7}$, con aval o garantía solidaria, enmarcados dentro de las líneas de microcréditos del Banco dirigidas al fortalecimiento de los pequeños negocios, a mujeres empresarias y a microcréditos agropecuarios. Dentro de este grupo se encuentran personas que han hecho uso por una o más veces del microcrédito y su vinculación está fundamentada en la argumentación de Henríquez (2009), para quien el uso intensivo del microcrédito potencia los resultados encontrados en los beneficiarios.

\subsection{Estimación de Propensity Score Matching (PSM)}

Con base en las apreciaciones conceptuales del apartado anterior y en consideración a la naturaleza no aleatoria de los datos para observar el efecto de una política de micro-financiamiento sobre el bienestar de un individuo atribuible en este caso a la participación en el programa de microcrédito rural ofertado por el Banco Agrario seccional Tunja, es clave buscar y construir un grupo de comparación válido (contrafactual) para calcular el ATT (microcrédito) sobre los beneficiarios, en términos de las variables resultado a través de emparejamientos (Matching) para beneficiarios y controles, como se explica en Aedo (2005), y mediante técnicas estadísticas basadas en características observables, para lograr que una unidad beneficiaria se identifique con otra no beneficiaria que posee características muy similares.

El método de emparejamiento (Matching) para estimar el ATT del microcrédito mediante el PSM se efectúa en dos etapas. En la primera etapa se estima el Propen-

7 En 2016 el salario mínimo era de \$689.455. 
sity Score (PS) que indica la probabilidad de participar en el programa, mediante un modelo de elección binaria $\left(\operatorname{logit}^{8}\right)^{8}$. En la segunda etapa, se realiza el análisis de emparejamiento que permite conocer el efecto neto de la política, mediante el cálculo del ATT; es decir, determinar si el microcrédito se ha constituido en una herramienta de alivio a la pobreza, a través de cambios en la dimensión económica: ingresos, activos fijos y activos productivos de los beneficiarios del programa (index _ ecomo).

Para lograr la estimación del ATT, se sigue un primer paso que consiste en estimar PS de la variable de tratamiento con una lista de covariables utilizando un modelo logit, el cual estratifica los hogares de acuerdo con bloques según el pscore obtenido, además, explora que la distribución de las variables observables sea la misma para los individuos beneficiarios y para los no beneficiarios. En razón a que es de interés el efecto que pueden generar los microcréditos sobre la dimensión económica: ingresos, activos fijos y activos productivos, el PS relaciona hogares con microcréditos con hogares sin microcrédito, que tienen una probabilidad similar sujeta a las características económicas, sociales y geográficas.

Para la estimación del modelo logit, se incluyó un conjunto de variables, tales como la variable tratamiento ( $t=1$ si es beneficiario del microcrédito, $t=0$ no beneficiario del microcrédito), con una lista de covariables que son explicativas de la variable binaria del tratamiento, entre ellas, el tipo de terreno que describe la propiedad del inmueble sobre el que desarrolla las actividades económicas (propio, familiar, arrendado, garantía sobre la propiedad); el destino del crédito es una variable que obedece a las líneas de crédito establecidas por el banco para tal fin (capital de trabajo, inversión, líneas especiales, asociativo); duración del crédito que para este caso no excede los 36 meses; el género de la persona y el número de hijos que integran el hogar.

En la tabla 1 se reportan algunas estadísticas descriptivas de las variables incluidas en el modelo. Para el logit, después de testear con diferentes covariables explicativas, se llega al valor-P del estadístico $\chi^{2}(0,000)$ que indica que el coeficiente Pseudo- $\mathrm{R}^{2}$ es válido en 0,2618 , un ajuste del $\mathrm{R}^{2}$ con un parámetro bajo; sin embargo, como se alude en Blundell, Sianesi y Dearden (2005), si el pseudo R2 resulta bajo antes de que se efectúe el emparejamiento (matching), lo que está señalando es evidencia de que los grupos beneficiarios y controles tienen parámetros comparables, o se tiene la certeza de que estos están balanceados, es decir, son lo suficientemente similares como para asemejarse a grupos que hubiesen sido escogidos de forma aleatoria, como se indica en Sánchez (2015).

8 Se manifiesta por parte de los autores que la base de datos del modelo de elección binaria (logit), puede ser solicitada por los investigadores que tengan interés en ella al correo: gloriamercedes.manrique@uptc.edu.co 
Tabla 1. Regresión logística para PSM

\begin{tabular}{|c|c|c|c|c|c|c|c|}
\hline \multicolumn{2}{|c|}{$\begin{array}{c}\text { Logistic regression } \\
\text { Log Likelihood }=63,531092\end{array}$} & & & \multicolumn{2}{|c|}{$\begin{array}{l}\text { Number of obs } \\
\text { LR chi2 (5) } \\
\text { Prob > chi2 } \\
\text { Pseudo R2 }\end{array}$} & \multicolumn{2}{|c|}{$\begin{array}{l}128 \\
45,07 \\
0,000 \\
0,2618\end{array}$} \\
\hline MicroCredito & Coef. & Std. Err. & z & $\mathrm{P}>|z|$ & $195 \%$ & nf. Int & val] \\
\hline TipoTerreno & 0,6654582 & 0,2450383 & 2,72 & 0,007 & & 5192 & 1,145724 \\
\hline DestinoCredito & $-0,6310632$ & 0,1387931 & $-4,55$ & 0,000 & $-0,9$ & 9927 & $-0,3590338$ \\
\hline Duracion & $-0,5662473$ & 0,2833505 & $-2,00$ & 0,046 & -1 , & 1604 & $-0,0108906$ \\
\hline Genero & $-1,4879$ & 0,5832737 & $-2,55$ & 0,011 & -2 , & 1095 & $-0,3447048$ \\
\hline Hijos & 0,0914981 & 0,1352592 & $-0,68$ & 0,499 & -0 , & 3605 & 0,3566013 \\
\hline _ cons & 2,383774 & 1,267363 & 1,88 & 0,060 & $-0,10$ & 112 & 4,86776 \\
\hline
\end{tabular}

** Significativo al 5\%

\section{Fuente: elaboración propia}

Como medida complementaria de la bondad de ajuste del modelo logit se presenta la estimación de las pruebas de sensibilidad y especificidad. En la tabla 2, se determina la probabilidad correcta de los participantes en el GC y GT o el acercamiento del modelo a la realidad.

Para GT (+;D) la ecuación clasificó 63 beneficiarios del microcrédito pertenecientes a este grupo que representa una sensibilidad del modelo del $81,82 \%$ indicando la probabilidad de calificar de manera acertada a un beneficiario del microcrédito que forma parte del GT dentro de su propio grupo. Las diferencias $(18,18 \%)$ corresponden al error tipo I9.

Para el GC (-; D ), se clasificaron acertadamente 32 no beneficiarios del microcrédito, lo que señala una especificidad del 62,75\% y permite considerar un individuo del GC dentro de su propio grupo. Las diferencias (37,25\%), indican error tipo II $^{10}$.

La especificación del PS es correcta, y la clasificación de controles y beneficiarios es acertada en un $74,22 \%$, resultados que conllevan a disminuir el sesgo de selección y producen estimaciones más confiables. En el caso del estudio esto puede obedecer a que se usaron criterios considerados en Heckman, Ichimura y Todd (1998)

\footnotetext{
9 Error I: Clasificar a un beneficiario del microcrédito como no integrante del grupo de tratamiento del programa, siendo de este.

${ }_{10}$ Error II: Clasificar a un beneficiario de microcrédito como participante del grupo de control siendo que pertenece al grupo de tratamiento.
} 
aludidos a utilizar la misma fuente de datos ${ }^{11}$ para participantes y no participantes; de esta forma se asegura que las variables X fueron medidas bajo los mismos parámetros y significan lo mismo en ambas muestras: un buen control de las variables observables que estén afectando la participación en el programa (asumiendo que la selección no observada puede ser limitada).

Tabla 2. Distribución estimada de la variable de tratamiento antes del Matching

\begin{tabular}{|c|c|c|c|}
\hline \multirow{3}{*}{\begin{tabular}{|l} 
Logistic model for \\
Classified
\end{tabular}} & \multicolumn{3}{|c|}{ MicroCredito } \\
\hline & \multicolumn{2}{|c|}{ True } & Total \\
\hline & $\mathrm{D}$ & $\sim D$ & \\
\hline+ & 63 & 19 & 82 \\
\hline- & 14 & 32 & 46 \\
\hline Total & 77 & 51 & 128 \\
\hline \multicolumn{4}{|c|}{ Classified + if predicted $\operatorname{Pr}(\mathrm{D})>=.5$} \\
\hline \multicolumn{4}{|c|}{ True $\mathrm{D}$ defined as MicroCredito $=\mathrm{O}$} \\
\hline Sensitivity & \multicolumn{2}{|c|}{$\operatorname{Pr}(+\mid D)$} & $81,82 \%$ \\
\hline Specificity & \multicolumn{2}{|c|}{$\operatorname{Pr}(-\mid \sim D)$} & $62,75 \%$ \\
\hline Positive predictive value & \multicolumn{2}{|c|}{$\operatorname{Pr}(\mathrm{D} \mid+)$} & $76,83 \%$ \\
\hline Negative predictive value & \multicolumn{2}{|c|}{$\operatorname{Pr}(\sim \mathrm{D} \mid-)$} & $69,57 \%$ \\
\hline False + rate for true $\sim D$ & \multicolumn{2}{|c|}{$\operatorname{Pr}(+\sim D)$} & $37,25 \%$ \\
\hline False - rate for true $\mathrm{D}$ & \multicolumn{2}{|c|}{$\operatorname{Pr}(-\mid \mathrm{D})$} & $18,18 \%$ \\
\hline \multicolumn{3}{|c|}{ False + rate for classified $+\operatorname{Pr}(\sim D \mid+)$} & $23,17 \%$ \\
\hline \multicolumn{3}{|c|}{ False - rate for classified $-\operatorname{Pr}(D \mid-)$} & $30,43 \%$ \\
\hline \multicolumn{3}{|l|}{ Correcly classified } & $74,22 \%$ \\
\hline
\end{tabular}

** Significativo al 5\%

Fuente: elaboración propia

De igual forma, se incluye un análisis visual del test de igualdad de las distribuciones de densidad de la probabilidad estimada de participación con soporte común (gráfico 2). Para mayor solapamiento o intersección en la distribución de observables entre el grupo de control y beneficiarios de la dimensión económica en la prueba, se excluyeron dieciséis observaciones dado que se aduce presencia de áreas donde el PS de algún grupo es cero, es decir, se excluyeron aquellas que no pueden emparejarse porque no existe contrafactual para ellos como lo explican Rosenbaum y Rubin (1983).

${ }^{11}$ El grupo control fue seleccionado de la base de datos aportada por el Banco Agrario y se tuvieron en cuenta personas que manifestaron intensión de acceder a esta línea de crédito pero que finalmente no lo hicieron y personas con proximidad geográfica y dedicación económica similar a la de los tratados. 
Si bien la gráfica no es simétrica, existe región de soporte común lo que implica que en la estimación solo se utilizan individuos del grupo control que tengan probabilidades de participación $\mathrm{p}(\mathrm{X})$ similares a las de participación de los beneficiarios, como se considera en Tamayo (2012). De esta forma, utilizar p(X) como método de emparejamiento es un atajo efectivo que permite obtener estimadores consistentes, de acuerdo con Gertler et al. (2011).

Gráfico 2. Test de igualdad de distribuciones para dimensión económica

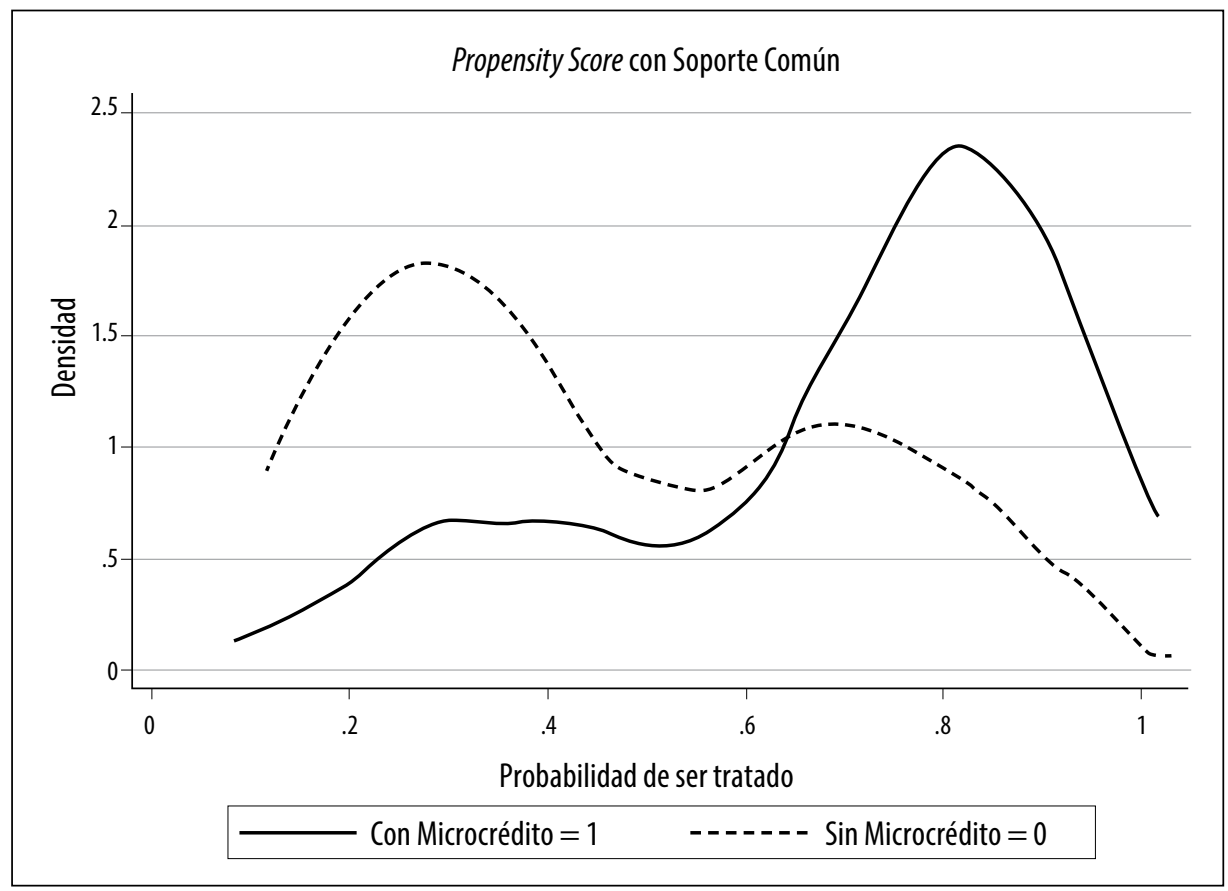

Fuente: elaboración propia

Estimado el PS, la segunda etapa consiste en hallar los emparejamientos (matching), para determinar el ATT producido por el microcrédito en la dimensión económica de quienes accedieron al mismo sobre las variables resultado: ingresos e índex-econo; para este último del cálculo por componentes principales se obtuvo:

$$
\boldsymbol{a}_{j}=\left|\begin{array}{r}
-0,4803 \\
1,6659 \\
0,4889 \\
\ldots \ldots . . \\
0,3159
\end{array}\right|
$$


Del producto $a_{j}^{\prime} z$ resultan 128 magnitudes para el índex-econo, una por cada encuestado que es la variable resultado que se utiliza para medir el impacto del microcrédito. El valor del índice osciló entre -0,1904 y 1,8389; dada la normalización de las variables en el inductor, algunas observaciones pueden tomar valores negativos y transmitir el signo al índice por cuanto algunos individuos carecen de los activos productivos.

Siguiendo con la estimación de los emparejamientos (matching), al no contar con muestras grandes y acudiendo a la recomendación de Bernal y Peña (2011) en el sentido de usar más de un método para comparar solo parejas idénticas y asegurarse que mediante técnicas con escaneos y aproximaciones diferentes en zona de soporte común se llegue a conclusiones significativas desde diferentes alternativas y así robustecer los resultados, en el estudio se usaron varios métodos de emparejamiento: vecino más cercano, radius (calipers), kernel, estratificación.

El algoritmo vecino más cercano compara a cada individuo beneficiario del microcrédito con un individuo no beneficiario de este que tiene el PS más próximo; radius (calipers) se estima con la selección de una pareja dentro de una vecindad delimitada de beneficiarios y no beneficiarios del programa: si el radio es de menor tamaño reduce la posibilidad de emparejamientos, pero mejora la calidad de los mismos; kernel es el criterio por medio del cual se compara cada beneficiario del crédito con una media ponderada de los resultados de todas las unidades del grupo de control o no beneficiarios, utilizando las mayores ponderaciones para las unidades con PS más parecido al comparado; estratificación agrupa los PS en categorías de un determinado percentil, de manera que en cada uno de ellos se encuentren los individuos que tienen un índice de propensión semejante y pueden ser comparables.

Todos los cálculos parar el PSM, se ha efectuado utilizando software estadístico Stata.

\section{RESULTADOS}

\subsection{Variable resultado ingresos}

En la tabla 3 se presentan los resultados obtenidos para la variable ingreso ${ }^{12}$. El cálculo de los emparejamientos (matching) al 95\% de confianza muestra un impacto positivo diferente de cero referido al ATT, en términos de las pruebas vecino más cercano, radius (calipers) y estratificación.

\footnotetext{
${ }_{12}$ La variable se interpreta como cambios en el nivel de ingresos del grupo beneficiario, atribuidos al microcrédito, que pueden contribuir a la disminución de la pobreza.
} 
Tabla 3. Resumen estimaciones efectuadas en el análisis de impacto para la variable Ingresos

\begin{tabular}{|l|c|c|c|l|}
\hline \multicolumn{1}{|c|}{ Método Emparejamiento } & $\begin{array}{c}\text { Efecto del } \\
\text { tratamiento(ATT) }\end{array}$ & Error Estándar & t-Sudent & Resultado Estadístico \\
\hline Vecino más Cercano & 0,4677 & 0,1384 & 3,38 & Significativo \\
\hline Radius (calipers) & 0,5286 & 0,1907 & 2,77 & Significativo \\
\hline Kernel & 0,2540 & 0,1355 & 1,87 & No Significativo \\
\hline Estratificación & 0,3620 & 0,0840 & 4,32 & Significativo. \\
\hline
\end{tabular}

** Significativo al 5\%

\section{Fuente: elaboración propia}

Los emparejamientos que alcanzaron significancia estadística: vecino más cercano, radius (calipers) permitieron determinar que los beneficiarios del microcrédito incluidos dentro del GT, tuvieron un ATT entre el 0,3620 y el 0,5286, lo que aporta la posibilidad de incrementar sus ingresos en un rango entre 36,20\% y 52,86\% más en relación con los individuos del GC.

En la prueba Kernel, aunque hay evidencia de un efecto positivo, en relación con el ATT no se alcanza nivel de significancia estadística al 95\% $(t=1.87)^{13}$, lo que implica que bajo este emparejamiento los beneficiarios del microcrédito no logran un aumento en sus ingresos.

Para mayor apreciación del impacto del microcrédito sobre los ingresos de los beneficiarios, y teniendo en cuenta que la robustez del resultado es afectada por la no significancia estadística de la prueba Kernel, se analizó el promedio global del efecto ATT en las pruebas aplicadas, y se encontró que los ingresos del GT crecieron un $29,69 \%$ más respecto al grupo control.

El resultado obtenido es relevante, en razón a que el nivel de pobreza en áreas rurales es superior un 16,77\%, frente a las áreas urbanas, de acuerdo con la información estadística presentada por el DANE (2016). Los aportes del microcrédito, en el área geográfica donde se aplicó el estudio, pueden entrar a categorizarse como

${ }^{13}$ Desde un punto de vista teórico en la metodología utilizada PSM, la baja significancia estadística al realizar los emparejamientos se puede presentar por el tamaño reducido de la muestra, en este estudio el segmento de microcréditos que se trabajó es una línea relativamente nueva en el Banco y, a la fecha de corte de la encuesta, su volumen de colocación de este microcrédito es bajo. Aunado a lo anterior la investigación se adelantó en Tunja y Samacá, siendo un universo pequeño en relación con los municipios atendidos por el banco. Se precisa, que después de la revisión en campo del portafolio del Banco Agrario, y a pesar de las limitantes anteriores, se determinó trabajar sobre el mismo, dado que es una de las líneas crediticias que ha sido implementada con muchas similitudes a Grameen y fundada en experiencias de banca para pobres como BancaSol Perú, Agroamigo Brásil. 
Impacto del microcrédito sobre la pobreza rural en los municipios de Tunja y Samacá, Colombia

medios $^{14}$, pero proporcionan evidencia para atribuir mejoras en el ingreso de los beneficiarios, lo que sugiere que las personas que pueden acceder al microcrédito son menos vulnerables a tener ingresos por debajo de la línea de pobreza.

\subsection{Resultados índex-econo}

Los emparejamientos vecino más cercano, kernel y estratificación, con un nivel de confianza del 95\%, para el índex-econo compuesto por activos fijos y productivos, relacionan efectos positivos del microcrédito, que se presentan en la tabla 4.

Tabla 4. Resumen estimaciones efectuadas en el análisis de impacto para el índex-econo

\begin{tabular}{lccccl}
\hline Método Emparejamiento & $\begin{array}{c}\text { Efecto del } \\
\text { tratamiento }\end{array}$ & $\begin{array}{c}\text { Error } \\
\text { Estándar }\end{array}$ & t-Student & $\begin{array}{c}\text { Porcentaje de } \\
\text { Impacto }\end{array}$ & $\begin{array}{c}\text { Resultado } \\
\text { Estadístico }\end{array}$ \\
\hline Vecino más Cercano & 0,3868 & 0,1583 & 2,44 & $13,92 \%$ & Significativo \\
Radius (calipers) & 0,2164 & 0,1481 & 1,46 & - & No Significativo \\
Kernel & 0,4728 & 0,1155 & 4,09 & $15,22 \%$ & Significativo \\
Estratificación & 0,4970 & 0,0860 & 5,78 & $17,89 \%$ & Significativo \\
\hline
\end{tabular}

** Significativo al 5\%

Fuente: elaboración propia

El emparejamiento estratificación con significancia estadística $t=5,78$ evidencia los mejores resultados del impacto del microcrédito en el GT. El ATT reflejó un 0,4970, que representa el 17,89\% de aumento de activos dedicados a mejorar los sistemas productivos de los beneficiarios del microcrédito. Los resultados de esta prueba son confirmados por las estimaciones logradas en los algoritmos kernel y vecino más cercano que con el 0,4723 y el 0,3868 e indican mayores tenencias de activos (maquinaria, herramientas y semovientes) en los beneficiarios, y representan un cambio porcentual entre el 13,92\% y 15,22\%.

En la técnica radius (calipers) no hay evidencia estadística significativa al 5\%, lo que indica que el microcrédito no ha permitido al GT adquirir activos con fines productivos. No obstante, es importante tener en cuenta que en la metodología aplicada, el emparejamiento radius (calipers) define una vecindad delimitada y, por

\footnotetext{
${ }^{14}$ Como lo explica Cohen (1998), en su estudio Statistical power analysis for the behavioral, un efecto puede precisarse como: trivial, pequeño, mediano o grande en relación con el efecto obtenido. Aunque los TE (efecto tratamiento), son difíciles de interpretar y su estimación acude a diversos métodos estadísticos, la literatura ha convergido a un paso tentativo para establecer ciertas convenciones, que a juicio de los investigadores revelen, de acuerdo al objeto de estudio, un acercamiento a la realidad percibida desde lo cualitativo.
} 
lo tanto, restringe las diferencias que debe haber en el PS para hacer el emparejamiento. Si la dimensión del vecindario (el radio) es demasiado pequeña es posible que algún participante del grupo tratado no encuentre su correspondiente control (en muestras pequeñas el radio puede acotarse más y es difícil encontrar efectos medios) como se sustenta en Vélez, Egurrola y Jaimes (2013).

De otra parte, desde el punto de vista teórico, este índice cobra vital importancia si se tiene en cuenta que una de las premisas básicas con que se concede el microcrédito es generar dinámicas de producción e inversión. Por tanto, los resultados de los algoritmos relacionados con el análisis del ATT, aunque un poco condicionados por la no significancia de la prueba radius (calipers), en su mejor magnitud (emparejamiento estratificación), muestran un grado de efecto del programa en los tratados del 17,89\% o medio, de acuerdo con Cohen (1998), es decir, que las personas que participan del programa de microcréditos rurales ofrecidos por el Banco Agrario en Tunja y Samacá han presentado cambios en su estructura productiva y en su nivel de tecnificación de labores ; aunque moderados, pueden estar coadyuvando a la transformación de la productividad diaria para fortalecer el patrimonio de los tratados, y se espera sean sostenibles, a fin de atenuar los shock que enfrentan los pobladores rurales por cambios en los términos de intercambio y externalidades como cambios climáticos que repercuten en el bienestar de las personas como se sustenta en Sen (1985).

En refuerzo a la tesis anterior, estudios del programa de microcrédito en Chile y Brasil desarrollados por Aroca (2002) abordan las microcréditos rurales, con un grado de similitud alto, en condiciones de lo estudiado en la presente investigación; con muestras analizadas en bancos y organizaciones no gubernamentales brasileñas y chilenas, han llegado a las conclusiones en las que la acumulación de activos utilizados en labores de producción, se vio incrementada en un 20\% en el grupo tratado, lo que significó para los investigadores una evolución media en las mejoras de los sistemas de producción de los individuos que, de ser sostenida en el tiempo, puede considerarse una medida efectiva contra la reducción de la pobreza.

\section{CONCLUSIONES Y RECOMENDACIONES}

Los cálculos para los emparejamientos (matching): vecino más cercano, radius calipers, kernel y estratificación permitieron determinar que hay efectos positivos causados por la participación en el programa de microcrédito ofertado por el Banco Agrario (seccional Tunja, Colombia), sobre la dimensión económica de los beneficiarios, es decir, el acceso al financiamiento provocó cambios en los ingresos, los 
Impacto del microcrédito sobre la pobreza rural en los municipios de Tunja y Samacá, Colombia

activos fijos y productivos que pueden indicar mejoras en la dotación de capital para el GT y un paliativo contra la pobreza.

Para la variable ingreso, el análisis de los datos en tres de las cuatro pruebas indicó resultados estadísticamente significativos. El ATT de los tratados sobre el GT fue del 29,69\%, lo que indica una condición de menor vulnerabilidad frente a estados de pobreza monetaria. Estos resultados concuerdan con las investigaciones de Zaman (1999) y Yunus (2008) las cuales demuestran mejoras en los ingresos para las personas que han accedido al microcrédito.

Respecto al índex-econo, la mayoría de las pruebas indicaron significancia estadística, lo que aduce efecto positivo sobre los beneficiarios del microcrédito; de esta forma se pudo llegar a una verificación de lo planteado en el estudio, en razón a que se pudo inferir que el microcrédito contribuye a mejorar las dotaciones productivas de los individuos, es decir, acumulación de capital. Sin embargo, estos resultados deben tomarse con cautela, pues su nivel de impacto es medio y se necesita de una sostenibilidad del mismo en el tiempo para pretender que las condiciones de bienestar de los evaluados se modifiquen de manera sustancial.

Cabe precisar que las evidencias obtenidas y analizadas en la dimensión económica no pueden considerarse de forma estricta, con fundamento en que las estimaciones aquí presentadas no están exentas de problema de sesgo, frente a un método semiparamétrico como el PSM. Si bien, respetando lo propuesto por la metodología, para mayor ajuste de la zona de traslape se excluyeron las unidades de la muestra no aptas para el PSM y los participantes y no participantes son de una misma zona geográfica como lo sugiere Ravallion y Chen (1997), esto no aísla en su totalidad los problemas de sesgo que puedan presentarse.

El ejercicio de estimación mostrado debe ser tomado en una medida adecuada a su contexto; el programa evaluado puede presentar limitaciones referidas a la captura total del efecto dado que se hace en un área geográfica pequeña, económica y socialmente diferente a otras, aunque puede servir de instrumento de apertura para que se replique de modo posterior bajo la misma metodología en busca de nuevos resultados que permitan seguir confirmando la importancia del microcrédito como herramienta de mitigación de la pobreza; en concordancia con lo que han sido los fines y alcances del Banco Grameen en Bangladesh, donde los beneficiarios obtienen una disminución en las tasas de interés y acceso a nuevos recursos cuando alcanzan como producto de los excedentes del préstamo: mejoras alimentarias, de vivienda, agua potable y educación. 


\section{BIBLIOGRAFÍA}

Aedo, Cristián (2005). Evaluación de Impacto. En: Cepal-Serie Manuales, No. 47, noviembre, 78p.

Álvarez, Jhon Jairo (2013). Evaluación del impacto del microcrédito en la Cooperativa de Ahorro y Crédito Colanta. Para optar el título de Magister en Ingeniería Administrativa. Maestría en Ingeniería Administrativa, Universidad Nacional, Colombia, 143p.

Armendaríz, Beatriz y Morduch, Jonathan (2005). The Economics of Microfinance. Massachusetts Institute of Technology, Estados Unidos de América, 257p.

Aroca, Patricio A. (2002). Microcrédito, evaluación de impacto. Casos: Brasil y Chile. En: IDEAR, Universidad Católica del Norte, mayo, p. 2-24.

Arun, Thankom; Imai, Katsushi y Sinha, Frances (2006). Does the Microfinance Reduce Poverty in India? Propensity Score Matching based on a National-level Household Data. En: Development Economics and Public Policy, Working Paper Series, No.17, septiembre, 31p.

Bernal, Raquel y Peña, Ximena (2011). Guía Práctica para la evaluación de impacto. Ediciones Uniandes: Bogotá, Colombia, 336p.

Blundell, Richard; Sianesi, Barbara y Dearden, Lorraine (2005). Evaluating the effect of education on earnings: models, methods and results from the National Child Development Survey. En: Journal of the Royal Statistical Society Series A, Vol. 168, No. 3, julio, p. 473-512. DOI: 10.1111/j.1467-985X.2004.00360.x.

Campbell, Donald T. y Stanley, Julian C. (1995). Diseños experimentales y cuasiexperimentales en la investigación social. Amorrortu editores; Buenos Aires, Argentina, 79p.

Carreño, Nubia Stella; Hernandez, Hugo Alfonso y Méndez, Jhon Alexander (2011). Microcrédito y bienestar: una evaluación empírica. En: Sociedad y economía, No. 21, septiembre, p.195-220.

Cohen, Jacob (1998). Statistical Power Analysis for the Behavioral Sciences. Lawrence Erlbaum Associates: New Jersey, USA, 87p.

Copestake, J.; Dawson, P.; Fanning, J.P.; McKay, A. y Wright-Revolledo, K. (2005). Monitoring the diversity of the poverty outreach and impact of microfinance: a comparison of methods using data from Peru. En: Development Policy Review, Vol. 23, No. 6, November, p. 703-723. Doi:http://dx.doi.org/10.1111/j.1467-7679.2005.00309.x.

Cotler, Pablo y Rodríguez-Oreggia, Eduardo (2010). Microfinanzas y la tenencia de activos no financierosen México. En: Investigación Económica, Vol. 69, No. 274, octubre-diciembre, p. 63-86.

DANE -Departamento Administrativo Nacional de Estadística - (2016). Pobreza Monetaria y Mul tidimensional en Colombia 2016. Boletín Técnico, 43p.

Filmer, Deon y Pritchett Lant H. (2001). Estimating Wealth Effects without Expenditure Data-or Tears: An Application to Educational Enrollments in States of India. En: Demography, Vol. 38, No. 1, febrero, p. 115-132.

Gertler, Paul J.; Martínez, Sebastián; Premand, Patrick; Rawlings, Laura B. y Vermeersch, Christel M. J. (2011). La evaluación de impacto en la práctica. Banco Internacional de Reconstrucción y Fomento/Banco Mundial: Washington DC, 229p. 
Impacto del microcrédito sobre la pobreza rural en los municipios de Tunja y Samacá, Colombia

Gibbons, Elizabeth y Garfield, Richard (1999). The Impact of Economic Sanctions on Health and Human Rights in Haiti 1991-1994. En: American Journal of Public Health, Vol. 89, No. 10, p. 1499-1504.

Heckman, James J.; Ichimura, Hidehiko y Todd, Petra (1998). Matching as an Econometric Evaluation Estimator. En: Review of Economic Studies, Vol. 65, No. 2, abril, p. 261-294.

Henríquez, Francisca (2009). Microcrédito y su impacto: un acercamiento con datos chilenos. En: Banco Interamericano de Desarrollo, Working Paper, No. OVE/WP-03/09, Washington, D.C., $30 p$.

Hossain, Mahabub (1988). Credit for alleviation of rural poverty: the Grameen Bank in Bangladesh. En: International Food Policy Research Institute, research report, No. 65, febrero, p. 1-96.

Hulme, David y Mosley, Paul (1996). Finance Against Poverty. Routledge: London, 221p.

Karlan, Dean y Zinman, Jonathan (2007). Observing Unobservables: Identifying Information Asymmetries with a Consumer Credit Field Experiment. En: Center for Global Development, Working Paper, No. 109, enero, 60p.

Khandker, Shahidur R. (1998). Figthing Poverty with Microcredit. Experience in Bangladesh. Word Bank: Washington, D.C., U.S.A., 219p.

Lacalle, Maricruz; Rico, Silvia y Durán, Jaime (2008). Estudio piloto de evaluación de impacto del programa de microcréditos de Cruz Roja Española en Ruanda. En: Revista de Economía Mundial, No. 19, enero, p. 83-104.

MkNelly, Barbara y Dunford, Christopher (1998). Impact of credir wirh education on morhers and rheir young children 's nutririon: Lower Pra Rural Bank Credir wirh Education Program in Ghana. En: Freedom From Hunger, Research Paper, No. 4, marzo, 72p.

MkNelly, Barbara y Dunford, Christopher (1999). Impacto de crédito con educación en las madres y en la nutrición de sus niños pequeños: programa CRECER de crédito con educación en Bolivia. En: Freedom From Hunger, Trabajo de Investigación, No. 5, marzo, 130p.

Moran, María Emilia y Gerrero, Alicia (2009). Evaluación ex-post del impacto del programa de microcrédito desarrollado por la Corporación Hogar de Cristo- Guayaquil. En: DSpace en ESPOL, febrero, 10p.

Morduch, Jonathan (1998). Income Smoothing and Consumption Smoothing. En: Journal of Economic Perspectives, Vol.9, No. 3, p. 103-114. DOI: 10.1257/jep.9.3.103.

Ravallion, Martín y Chen, Shaohua (1997). What Can New Survey Data tell us about Recent Changes in Distribution and Poverty? En: World Bank Economic Review, Vol. 11, No. 2, mayo, p.357-382. doi.org/10.1093/wber/11.2.357.

Rosenbaum, Paul R. y Rubin, Donald B. (1983). The Central Role of the Propensity Score in Observational Studies for Causal Effects. En: Biometrika, Vol. 70, No. 1, abril, p. 41-55.

Sánchez, Retana C. (2015). Evaluación de impacto de la educación sobre la pobreza en costa rica (un análisis para educación secundaria y post-secundaria). En: Ciencias Económicas, Vol. 33, No. 2, noviembre, p. 1 16. DOI: http://dx.doi.org/10.15517/rce.v33i2.22608. 
Sen, Amartya (1985). Commodities and capabilities. Amsterdam: Elsevier Science: New York, USA, 232p.

Snow Douglas R. y Terry F. Buss (2001). Development and the Role of Microcredit. En: Policy Studies Journal, Vol. 29, No. 2, mayo, p.296-307. DOI: 10.1111/j.1541-0072.2001.tb02093.x.

Tamayo, Laura Juliana (2012). Distribución regional de las regalías directas en Colombia y sus efectos sobre el desarrollo socioeconómico entre regiones receptoras y no receptoras de regalías. Tesis para oprtar el título de Magister en Economía. Maestría en Economía, Pontificia Universidad Javeriana, Bogotá, 74p.

Vélez, Marcela; Egurrola, Jorge y Jaimes Fabián (2013). Ronda clínica y epidemiológica. Uso de la puntuación de propensión (propensity score) en estudios no experimentales. En: Iatreia, Revista Médica Universidad de Antioquia, Vol. 26, No. 1, enero, p. 95-101.

Woller, Gary; Dunford Christopher y Woodworth, Warner (1999). Where to microfinance? En: International Journal of Economic Development, Vol. 1, No. 1, p. 29-64.

Yunus, Muhammad (2008). Creating a World without Poverty, Social Business and the Future of capitalism. En: Global Urban Development, Vol. 4, No. 2, noviembre, p. 1-18.

Yunus, Muhammad (2010). Muhammad Yunus y su visión de la pobreza. Conferencia Universidad Nacional Mayor de San Marcos, Perú, septiembre, 3p.

Zaman, Hassan (1999). Assessing the Impact of Micro-credit on Poverty and Vulnerability in Bangladesh. En: Policy Research Working Papers, No. 2145, julio, p.7-54. Doi.org/10.1596/1813 9450-2145. 


\section{ANEX0}

\section{ENCUESTAS PARA BENEFICIARIOS DE MICROCRÉDITO DEL BANCO AGRARIO - MUNICIPIOS DE TUNJA Y SAMACÁ}

La presente encuesta es de carácter académico y confidencial, está autorizada por la entidad, por lo que agradecemos mucho su objetividad en las respuestas.

\section{INFORMACIÓN GENERAL DEL PRODUCTO ADQUIRIDO POR EL CLIENTE}

La finca donde usted desempeña sus actividades productivas es:

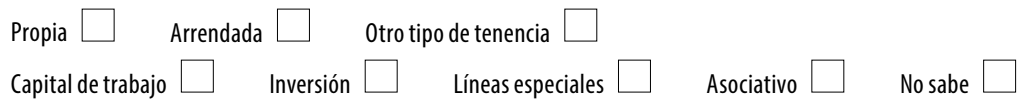

Usted ha solicitado crédito por:

Primera vez $\square \quad$ Una o más veces

Años de duración de crédito

Actividad en la que tradicionalmente se

El crédito le ha permitido diversificar su actividad agropecuaria, es decir, desarrollar otra. ¿Cuál?

Los requisitos exigidos por la entidad para otorgar el crédito, le han parecido:

\begin{tabular}{|l|l|l|}
\cline { 2 - 3 } \multicolumn{1}{c|}{} & Sí & No \\
\hline Muy fáciles de cumplir & & \\
\hline Medianamente fáciles de cumplir & & \\
\hline Difíciles de cumplir & & \\
\hline
\end{tabular}

Los costos para cumplir con los trámites exigidos por la entidad financiera para otorgar el crédito, le han parecido:

\begin{tabular}{|l|l|l|}
\cline { 2 - 3 } \multicolumn{1}{l|}{} & Sí & No \\
\hline Muy altos & & \\
\hline Altos & & \\
\hline Medianamente altos & & \\
\hline Bajos & & \\
\hline Está de acuerdo con ellos & & \\
\hline
\end{tabular}

Observaciones:

El tiempo que ha transcurrido entre la solicitud y el desembolso del crédito, le ha parecido:

\begin{tabular}{|l|l|l|}
\cline { 2 - 3 } \multicolumn{1}{c|}{} & Sí & No \\
\hline Mucho tiempo & & \\
\hline Poco tiempo & & \\
\hline Adecuado & & \\
\hline
\end{tabular}


Edad

Género Hombre $\square$ Mujer $\square$

Estado civil Casado(a) $\square \quad$ Soltero $\square \quad$ Separado(a) $\square \quad$ Unión libre $\square$

No. de hijos

Nivel de educación del jefe de hogar Primaria $\square \quad$ Bachillerato $\square$ Universitario $\square$ 0tro:

\section{INFORMACIÓN RELACIONADA CON LOS INGRESOS - SITUACIÓN FINANCIERA}

Como usuario de crédito usted considera que sus ingresos:

Aumentaron $\square$ Son constantes $\square \quad$ Han disminuido $\square$

Usted tiene ahorros en:

Productos financieros: Cuenta de ahorros $\square \quad$ CDT $\square \quad$ Otros. ¿Cuáles?

INFORMACIÓN SOBRE ACTIVOS

Producto del crédito, usted ha podido acumular activos que mejoren su actividad productiva como:

\begin{tabular}{|l|l|l|l|l|l|}
\cline { 3 - 6 } \multicolumn{1}{l|}{} & Sí & No & & Sí & No \\
\hline Adquirir nuevos terrenos & & & Sistemas de riego & & \\
\hline Maquinaria & & & Semillas mejoradas & & \\
\hline Herramientas & & & Bovinos & & \\
\hline Caprinos & & & Porcinos & & \\
\hline
\end{tabular}

0tros, especifique:

INFORMACIÓN RELACIONADA CON SALUD

En su hogar han mejorado las condiciones alimentarias Sí $\square$ No $\square$

\begin{tabular}{|c|c|c|}
\hline & Sí & No \\
\hline $\begin{array}{l}\text { Considera usted que las condiciones de salud } \\
\text { derivadas de aumentos en los ingresos en su }\end{array}$ & & \\
\hline Ha podido tener acceso y pagar con recursos & & \\
\hline Medicina especializada & & \\
\hline Tratamientos odontológicos especializados & & \\
\hline
\end{tabular}

En caso de enfermedad de usted 0 algún miembro de su familia, está en capacidad de costear un tratamiento, de forma inmediata sin recurrir a subsidios: Sí $\square$ No $\square$ 


\begin{tabular}{|c|c|c|}
\hline & Sí & No \\
\hline \multicolumn{3}{|l|}{ Todos los miembros de su hogar entre 1-4 años asiste a jardín o preescolar } \\
\hline \multicolumn{3}{|l|}{ Todos los miembros de su familia entre 5-12 años asisten a la escuela primaria } \\
\hline \multicolumn{3}{|l|}{ Todos los miembros de su familia entre 12-17 años asisten al colegio } \\
\hline \multicolumn{3}{|l|}{$\begin{array}{l}\text { Algunos de los miembros de su familia tiene acceso a educación primaria o secundaria en establecimientos privados, es decir, es } \\
\text { pagada de sus propios ingresos }\end{array}$} \\
\hline \multicolumn{3}{|l|}{ Los miembros de su familia con más de 17 años, han podido ingresar a la universidad } \\
\hline Falta de ingresos $\square \quad$ No quiere continuar estudiando $\square \quad$ 0tro. ¿Cuál? & & \\
\hline \multicolumn{3}{|l|}{ Usted u otros miembros de su hogar han podido adelantar capacitaciones en: } \\
\hline \multicolumn{3}{|l|}{ SENA } \\
\hline \multicolumn{3}{|l|}{ Institutos de formación para el trabajo } \\
\hline Otra. ¿Cuál? & & \\
\hline
\end{tabular}

Su vivienda cuenta con:

\begin{tabular}{|l|l|l|}
\cline { 2 - 3 } \multicolumn{1}{c|}{} & Sí & No \\
\hline Agua & & \\
\hline Sanitario & & \\
\hline Electricidad & & \\
\hline
\end{tabular}

Usted ha realizado mejoras en su vivienda, como resultado de mejores ingresos generados por el crédito, tales como:

\begin{tabular}{|l|l|l|}
\cline { 2 - 3 } \multicolumn{1}{l|}{} & Sí & No \\
\hline Pisos & & \\
\hline Paredes & & \\
\hline Instalaciones de duchas & & \\
\hline Aumentó el número de habitaciones & & \\
\hline Todos los miembros de su hogar duermen en cama & & \\
\hline
\end{tabular}

CAMBIOS EN LAS CONDICIONES DE VIDA

Producto de las mejoras obtenidas por el crédito usted ha podido comparar alguno de los siguientes activos:

\begin{tabular}{|l|l|l|l|l|l|l|l|l|}
\cline { 2 - 8 } \multicolumn{1}{l|}{} & Sí & No & & Sí & No & & Sí & No \\
\hline Radio & & & Estufa, a gas u otro combustible diferente a leña & & & Celular & & \\
\hline Televisor & & & Bicicleta & & & Lavadora & & \\
\hline Nevera & & & Moto & & & Automóvil & & \\
\hline
\end{tabular}


Gloria Manrique Joya - Maricela Ramírez - Félix Santos Varón

\begin{tabular}{|l|l|l|}
\cline { 2 - 3 } \multicolumn{1}{c|}{} & Sí & No \\
\hline Han experimentado cambios positivos en su forma de vestir & & \\
\hline Han podido disfrutar de paseos o vacaciones & & \\
\hline La visita al establecimiento bancario le ha permitido: & & \\
\hline Compartir con sus vecinos & & \\
\hline Hacer nuevos amigos & & \\
\hline Potenciar su poder de negociación & & \\
\hline Incrementar su autoestima & & \\
\hline Elegir de forma más autónoma sobre su negocio & & \\
\hline
\end{tabular}

Mil gracias 